\title{
Comprometimento Cardíaco na Síndrome de Sweet: Um Achado Raro numa Doença Rara
}

\author{
Myocardial Involvement in Sweet Syndrome: A Rare Finding in a Rare Condition
}

Luís Graça-Santos, ${ }^{1 \oplus}$ Katarina Kieselova, ${ }^{2}$ Fernando Montenegro-Sá, ${ }^{1 \oplus}$ Joana Guardado, ${ }^{2}$ João Morais ${ }^{2}$

Departamento de Cardiologia, Centro Hospitalar de Leiria, ${ }^{1}$ Leiria - Portugal

Departamento de Dermatologia, Centro Hospitalar de Leiria, ${ }^{2}$ Leiria - Portugal

\section{Introdução}

A Síndroma de Sweet (SS) é uma dermatose neutrofílica aguda febril caraterizada pela associação de febre, neutrofilia, lesões cutâneas eritematosas moles (pápulas, nódulos, placas), com histologia consistindo predominantemente em neutrófilos maduros localizados na derme superior. ${ }^{1}$ É uma condição rara com distribuição mundial e três formas de apresentação diferentes: idiopática; associada a malignidade e induzida por drogas. ${ }^{1-3}$ Manifestações extracutâneas podem ocorrer, mas o comprometimento cardiovascular é extremamente raro. ${ }^{1,2}$

\section{Caso clínico}

Homem de 41 anos procurou o serviço de urgência por febre ligeira e lesões cutâneas em agravamento desde há 48 horas. O paciente negou o uso de drogas, alergias conhecidas, histórico pessoal ou familiar de doença relevante, assim como contexto epidemiológico suspeito.

O paciente apresentou-se febril $\left(38,3^{\circ}\right)$ e a inspeção revelou pápulas e placas eritematosas, pseudovesiculadas e dolorosas na nuca, pescoço, ombros e braços, assim como nódulos subcutâneos eritematosos e dolorosos (tipo eritema nodoso) nas pernas (Figura 1). Restante exame objetivo sem alterações. O estudo laboratorial mostrou leucocitose ligeira (10800/uL) com 81,4\% de neutrófilos, velocidade de sedimentação $89 \mathrm{~mm} / \mathrm{h}$ (valor normal $(\mathrm{N})<10$ ) e proteína-C reactiva (PCR) 128,5 mg/L $(\mathrm{N}<5,0)$. Os eletrólitos e os testes de função hepática e renal eram normais.

Poucas horas após a admissão, o paciente manifestou desconforto torácico ligeiro em repouso. O eletrocardiograma mostrou ritmo sinusal a 58 por minuto com bloqueio atrioventricular do 10 grau e bloqueio incompleto do ramo direito. A troponina I (Tnl) foi 1,89ng/mL $(\mathrm{N}<0,05)$ e aumentou para 10,82ng/mL após seis horas. O segundo eletrocardiograma mostrou-se sobreponível. O ecocardiograma transtorácico (ETT) foi normal e demonstrou fração de ejeção ventricular esquerda

\section{Palavras-chave}

Síndrome de Sweet/fisiopatologia; Eritema Multiforme; Neutrófilos; Miocardite; Corticosteróides/uso terapêutico.

Correspondência: Luís Graça Santos •

Centro Hospitalar de Leiria - Rua Santo Andre Pousos. 2410-196, Leiria - Portugal E-mail: luismscp1@gmail.com

Artigo recebido em 13/04/2019, revisado em 27/07/2019, aceito em $18 / 08 / 2019$

DOI: https://doi.org/10.36660/abc.20190249

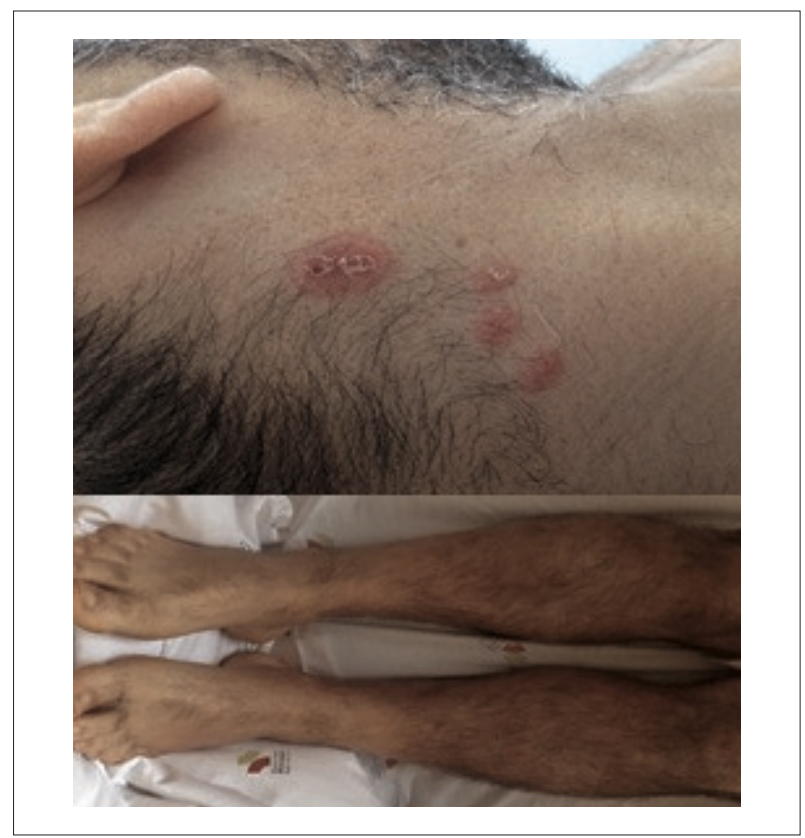

Figura 1 - Lesões cutâneas. Pápulas e placas eritematosas, pseudovesiculadas e dolorosas na nuca (topo); nódulos subcutâneos eritematosos e dolorosos nas pernas (inferior).

preservada (FEVE; 53\% pelo método Simpson biplano) sem alterações da cinética segmentar. Contudo, o strain longitudinal global (SLG) mostrou-se reduzido, especialmente à conta dos segmentos médio-basais, com os apicais relativamente poupados (Figura 2.A). A angiografia coronariana excluiu doença arterial coronariana (DAC) obstrutiva.

O paciente foi internado com o diagnóstico presuntivo de dermatose neutrofílica aguda febril. No segundo dia (D2), foi realizada biópsia cutânea e iniciada prednisolona (PDN) oral $1 \mathrm{mg} / \mathrm{kg} /$ dia tendo em conta a persistência da febre e das lesões cutâneas assim como o aumento da PCR (242 mg/L). Apesar do alívio total da dor torácica, a Tnl subiu para 15,01 $\mathrm{ng} / \mathrm{mL}$ no D2. Após o início de PDN, o paciente permaneceu apirético e os marcadores de inflamação e de necrose miocárdica regrediram. Testes laboratoriais complementares (proteinograma eletroforético, testes de autoimunidade, hormônios tireoidianos, hemoculturas, serologias) revelaramse normais. A análise histológica da pele mostrou edema subepitelial, infiltrado inflamatório na derme com predomínio polimorfonuclear, e ausência de vasculite (Figura 3). Desse modo, os critérios para o diagnóstico de $\mathrm{SS}^{3}$ foram satisfeitos e considerou-se elevada probabilidade de comprometimento 


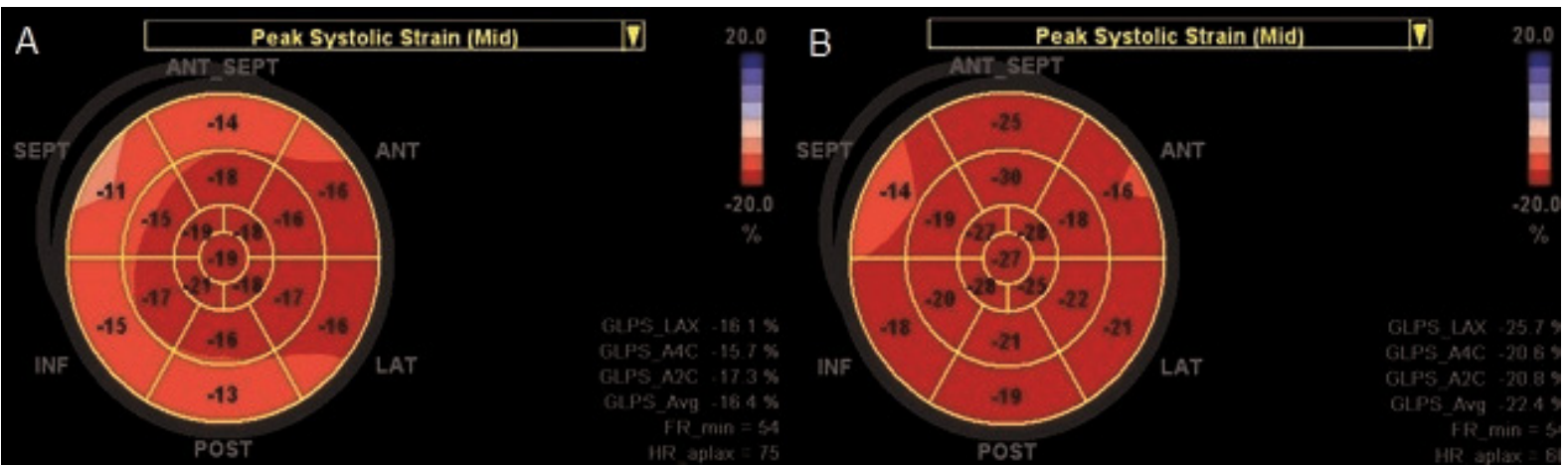

Figura 2 - Strain longitudinal global e segmentar; representação "olho-de-boi" (General Electric®). A) À admissão, o strain global está reduzido (-16,4\%); B) Três meses após o tratamento com corticosteroides, o valor normalizou $(-22,4 \%)$.

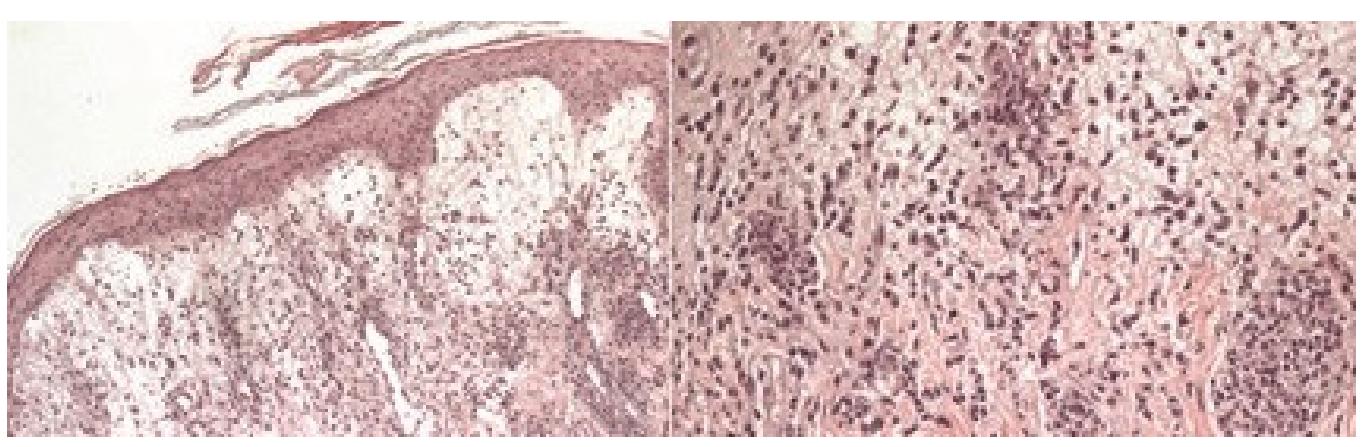

Figura 3 - Histologia da lesão cutânea cervical (coloração hematoxilina-eosina). Edema e infiltrado com predomínio de neutrófilos na derme (esquerda); Ampliação da região da derme mostrando linfócitos, histiócitos e ausência de vasculite (direita).

cardiovascular na forma de miocardite aguda (MA). ${ }^{3}$ Até D9, o paciente permaneceu apirético, as lesões cutâneas quase cicatrizaram, e os níveis de PCR e Tnl diminuíram (9,9 mg/L e $0,32 \mathrm{ng} / \mathrm{dL}$ respectivamente). Recebeu alta hospitalar sob PDN em redução progressiva.

Após quatro dias, o paciente apresentou-se completamente assintomático, sem lesões cutâneas e ambos os marcadores de inflamação e de necrose miocárdica normalizaram. No sexto dia após a alta, a ressonância magnética cardíaca (RMC) mostrou achados sugestivos de miocardite (Figura 4). A FEVE e SLG melhoraram para $63 \%$ e $-22,4 \%$, respectivamente, três meses após o tratamento (Figura 2.B). O paciente não desejou submeter-se a nova RMC.

Durante dois anos de seguimento, o paciente permaneceu assintomático e sem sinais ou sintomas de doença cardiovascular ou de malignidade.

\section{Discussão}

Os autores apresentam um caso no qual o diagnóstico de SS foi estabelecido pela presença de 2 critérios major e dois minor dos propostos por Driesch. ${ }^{4} \mathrm{O}$ tipo idiopático foi assumido devido à ausência de patologia maligna e do uso prévio de quaisquer fármacos. As manifestações extracutâneas podem ocorrer, particularmente em associação com malignidade. ${ }^{1,2} \mathrm{O}$ comprometimento cardiovascular é extremamente raro e, à luz do nosso conhecimento, apenas dois casos de miocardite foram reportados na variante idiopática. ${ }^{2,5,6}$ Ambas as manifestações tipicamente respondem aos corticosteroides. ${ }^{1}$

No caso descrito, o desconforto torácico transitório associado à elevação da Tnl levantou a suspeita de comprometimento cardiovascular. Quer a MA como o infarto agudo do miocárdio estão descritos como manisfestações cardiovasculares. ${ }^{2}$ A angiografia coronariana, que se mantém como o gold standard para o diagnóstico de $\mathrm{DAC}^{7}$ ou para sua exclusão na suspeita de $\mathrm{MA},{ }^{8}$ revelou-se normal. Existe alguma evidência de que a ecocardiografia bidimensional speckle tracking (2D-EST) pode ajudar a suportar o diagnóstico de MA uma vez que o strain longitudinal se correlaciona com a presença de fibrose e edema na RMC e de infiltração linfocitária na biópsia endomiocárdica (BEM). ${ }^{9-12}$ No caso relatado, a presença de SLG reduzido sobretudo à custa dos segmentos médio-basais, ao invés dos médio-apicais (padrão típico de DAC significativa), ${ }^{13}$ e a rápida resposta aos corticosteroides 


\section{Relato de Caso}

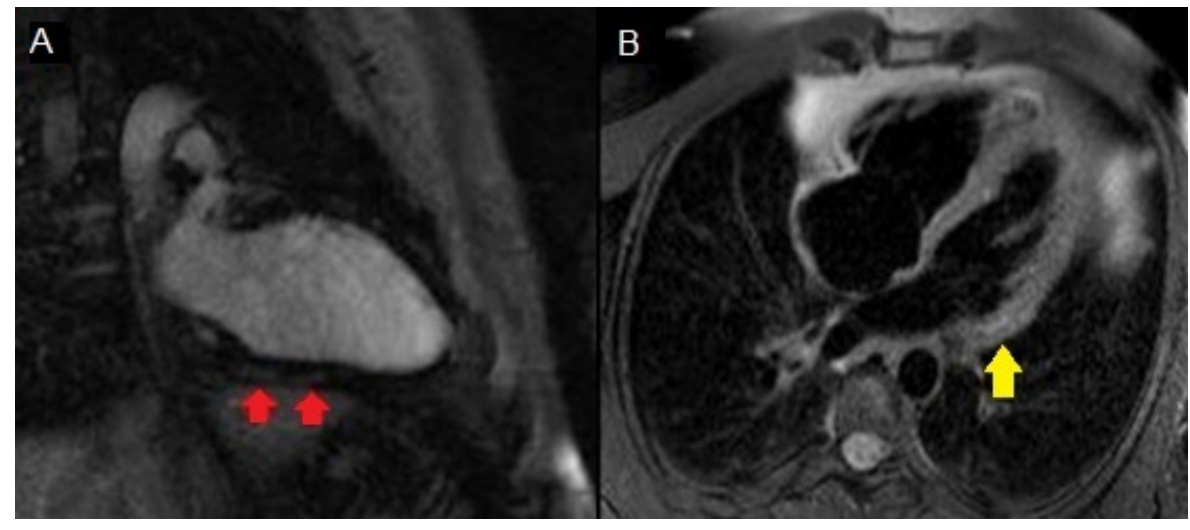

Figura 4 - Ressonância magnética cardíaca. A) Ponderação T1 mostrando ligeiro realce tardio de gadolínio na porção basal da parede inferior (setas vermelhas); B) Ponderação T2 apresentando regiões focais de intensidade de sinal aumentada sugestivas de discreto edema da parede inferolateral (seta amarela).

reforçaram a probabilidade de MA. Devido à estabilidade clínica e às conhecidas limitações da BEM, foi realizada RMC que sugeriu este diagnóstico de acordo com os critérios Lake-Louise. ${ }^{8,12}$ De fato, a RMC emergiu como ferramenta diagnóstica não invasiva e há evidência crescente de que novas técnicas, como o T1 e T2 mapping, podem melhorar a acuidade diagnóstica para MA e também ajudar na monitorização da doença. 8,14,15 Adicionalmente, o SLG normalizou três meses após o tratamento, enquanto o paciente permanecia assintomático.

\section{Mensagens finais}

O caso que apresentamos dá ênfase à importância de reconhecer a SS como uma rara mas possível causa de doença cardiovascular, entidade que deve ser precocemente identificada de modo a iniciar tratamento adequado.

No caso presente, o diagnóstico de MA foi altamente sugerido por modalidades imagiológicas não invasivas após a exclusão de DAC obstrutiva. À luz do nosso conhecimento, essa foi a primeira vez que a RMC foi usada para avaliar comprometimento miocárdico num doente com SS e também a primeira em que a análise por 2D-EST foi usada na monitorização evolutiva. Ambas as manifestações, cutâneas e

\section{Referências}

1. Cohen PR. Sweet's syndrome - a comprehensive review of an acute febrile neutrophilic dermatosis. Orphanet J Rare Dis. 2007 jul 26;2:34.

2. Villarreal-Villarreal CD, Ocampo-Candiani J, Villarreal-Martínez A. Sweet Syndrome: A Review and Update. Actas Dermosifiliogr. 2015;107(5):369-78.

3. Cohen PR, Kurzrock R. Sweet's syndrome revisited: a review of disease concepts. Int J Dermatol. 2003;42(10):761-78.

4. Von den Driesch P. Sweet's syndrome (acute febrile neutrophilic dermatosis). J Am Acad Dermatol. 1994;31(4):535-56.

5. DorenkampM, Weikert U, Meyer R, Schwimbeck PL, MorguetAJ. Heartfailure in acute febrile neutrophilic dermatosis. Lancet. 2003;362(9393):1374 cardiovasculares, regrediram completamente após tratamento com corticosteroides.

\section{Contribuiç̧ão dos autores}

Concepção e desenho da pesquisa e Obtenção de dados: Santos LG, Kieselova K; Análise e interpretação dos dados: Santos LG, Kieselova K, Sá FM, Guardado J; Redação do manuscrito: Santos LG, Sá FM, Morais JÁ; Revisão crítica do manuscrito quanto ao conteúdo intelectual importante: Guardado J, Morais JÁ.

\section{Potencial conflito de interesses}

Os autores declaram não haver conflito de interesses pertinentes.

\section{Fontes de financiamento}

O presente estudo não contou com fontes de financiamento externas.

\section{Vinculação acadêmica}

Não há vinculação deste estudo a programas de pós-graduação.

6. Yu WY, Manrriquez E, Bhutani T, Chaganti RK, Ruben BS, Schwartz BS, et al. Sweet heart: A case of pregnancy-associated acute febrile neutrophilic dermatosis with myopericarditis. JAAD Case Rep. 2014;1(1):12-4.

7. Montalescot G, Sechtem U, Achenbach S, Sechtem U, Andreotti F, Arden C, et al. 2013 ESC guidelines on the management of stable coronary artery disease: the Task Force on the management of stable coronary artery disease of the European Society of Cardiology. Eur Heart J. 2013;34(38):2949-3003.

8. Caforio AL, Pankuweit S, Arbustini E, Basso C, Gimeno-Blanes J, Felix SB, et al. Currentstate of knowledge on aetiology, diagnosis, management, and therapy of myocarditis: a position statement of the European Society of Cardiology Working Group on Myocardial and Pericardial Diseases. Eur Heart). 2013;34(33):2636-48. 
9. Leitman M, Vered Z, Tyomkin V, Macogon B, Peleg E, Copel L. Speckle tracking imaging in inflammatory heart diseases. Int J Cardiovasc Imaging. 2018;34(5):787-92.

10. Kostakou PM, Kostopoulos VS, Tryfou ES, Giannaris VD, Rodis IE, Olympios $C D$, et al. Subclinical left ventricular dysfunction and correlation with regional strain analysis in myocarditis with normal ejection fraction. A new diagnostic criterion. Int J Cardiol. 2018;259:116-21.

11. Løgstrup BB, Nielsen JM, Kim WY, Poulsen SH. Myocardial oedema in acute myocarditis detected by echocardiographic 2D myocardial deformation analysis. Eur Heart J Cardiovasc Imaging. 2016;17(9):1018-26.

12. Escher F, Kasner M, Kühl U, Heymer J, Wilkenshoff U, Tschope C, et al. New echocardiographic findings correlate with intramyocardial inflammation in endomyocardial biopsies of patients with acute myocarditis and inflammatory cardiomyopathy. Mediators Inflamm. 2013;2013:875420.

13. Carstensen HG, Larsen LH, Hassager C, Kofoed KF, Jensen JS, Mogelvang R. Association of ischemic heart disease to global and regional longitudinal strain in asymptomatic aortic stenosis. Int J Cardiovasc Imaging. 2015;31(3):485-95.

14. André F, Stock FT, Riffel J, Giannitsis E, Steen H, Scharhag J, et al Incremental value of cardiac deformation analysis in acute myocarditis: a cardiovascular magnetic resonance imaging study. Int J Cardiovasc Imaging. 2016;32(7):1093-101.

15. Roller FC, HarthS, Schneider C, Krombach GA. T1, T2 Mapping and Extracellular Volume Fraction (ECV): Application, Valueand Further Perspectives in Myocardial Inflammation and Cardiomyopathies. Rofo. 2015 Sep;187(9):760-70. 